\title{
High Power Lasers and Their New Applications
}

\author{
Yasukazu Izawa', Noriaki Miyanaga', Junji Kawanaka', and Koichi Yamakawa² \\ ${ }^{1}$ Institute of Laser Engineering, Osaka University Yamada-oka, Suita, Osaka 565-0871, Japan \\ ${ }^{2}$ Advanced Photon Research Center, Japan Atomic Energy Agency Umemidai, Kizugawa, Kyoto \\ 619-0215, Japan
}

(Received August 20, 2008 : revised September 11, 2008 : accepted September 11, 2008)

\begin{abstract}
Recent progress in high power lasers enables us to access a regime of high-energy-density and/or ultra-strong fields that was not accessible before, opening up a fundamentally new physical domain which includes laboratory astrophysics and laser nuclear physics. In this article, new applications of high-energy and ultra-intense laser will be reviewed.
\end{abstract}

Keywords : high power lasers; advanced lasers; laser fusion; high-energy density science, relativistic plasma; laser generation of energetic particles; laser driven nuclear processes

OCIS codes : (140.0140) Lasers and laser optics; (190.0190) Nonlinear optics: (350.2660) Fusion; (350.5400) Plasmas; (350.4990) Particles; (320.7110) Ultrafast nonlinear optics

\section{INTRODUCTION}

Progress in laser technology has made it possible to obtain $100 \mathrm{~kJ}$ in laser pulse energy and $1 \mathrm{PW}$ in laser peak power. In the near future, we will reach MJ and multi PW levels. Laser fusion research played an important role in developing high-energy and high-peak power lasers. By focusing such lasers we can create the energy density of $\sim 10^{12} \mathrm{~J} / \mathrm{cm}^{3}$ and the radiation intensity of $\sim 10^{21}$ $\mathrm{W} / \mathrm{cm}^{2}$, concentrated in a tiny plasma. The appearance of this new tool has a revolutionary significance in basic physics and also in industrial applications. New fields of science are emerging - laboratory astrophysics, and laser nuclear science. A number of new applications to material science, nuclear engineering, medical engineering and so on have been proposed. Here the present status and future prospect of laser fusion research, new industrial applications of high-energy and high-peak power lasers will be reviewed. And an advanced laser to realize such new applications will be discussed.

\section{PROGRESSES OF HIGH POXER LASERS AND LASER FUSION RESEARCH}

Developments of high power lasers have been acceler-

\footnotetext{
*Corresponding author: izawa@ile.osaka-u.ac.jp
}

ated by the laser fusion research. The progress in pulse energy of high power lasers is shown in Fig. 1. After the proposal of a central ignition scheme of laser fusion by the use of laser driven implosion in 1972, high-energy lasers such as OMEGA, GEKKO XII, SHIVA, NOVA and PHEBUS were developed during 1980's and 1990's. All these lasers are based on Nd:glass in a single shot operation and can deliver the pulse energy of $10^{\sim} 100$ $\mathrm{kJ}$ in $1 \sim 10 \mathrm{~ns}$ pulse duration. High-temperature compression up to $10 \mathrm{keV}$ and high-density compression up to $1 \mathrm{~kg} / \mathrm{cm} 3$ have been achieved by these lasers $[1,2]$. After that the laser fusion research moved into the new stage to demonstrate fusion ignition and burn. For this purpose MJ lasers are under construction by NIF (National Ignition Facility) [3, 4]and LMJ (Laser Mega Joule) [5] projects in USA and France, respectively.

After the invention of the chirped pulse amplification (CPA) technique, the peak intensity of the short pulse laser remarkably increased as shown in Fig. 2. A peak power of a petawatt (PW) has been achieved by Nd:glass $(\sim 500 \mathrm{~J}$ in $\sim 500 \mathrm{fs})[6,7]$ and Ti:sapphire $(\sim 15 \mathrm{~J}$ in $\sim 15 \mathrm{fs}$ ) [8] lasers and an exawatt (EW) laser is proposed [9]. Through such progresses, a new scheme of laser fusion, named fast ignition, became possible, where a laser fusion fuel pellet is first compressed by a high-energy laser and then the compressed fuel is heated up to ignition temperature by a PW laser. Feasibility of this scheme was demonstrated by the combination of GEKKO XII and 


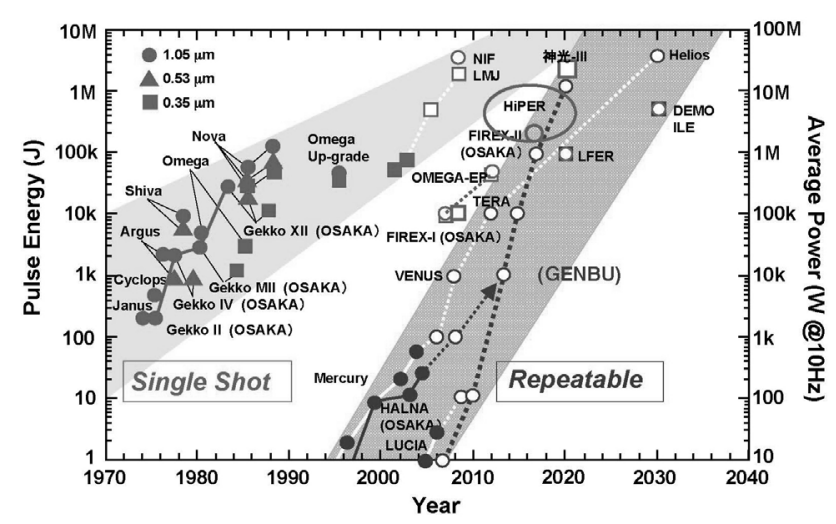

FIG. 1. Progress in high-energy lasers for laser fusion research.

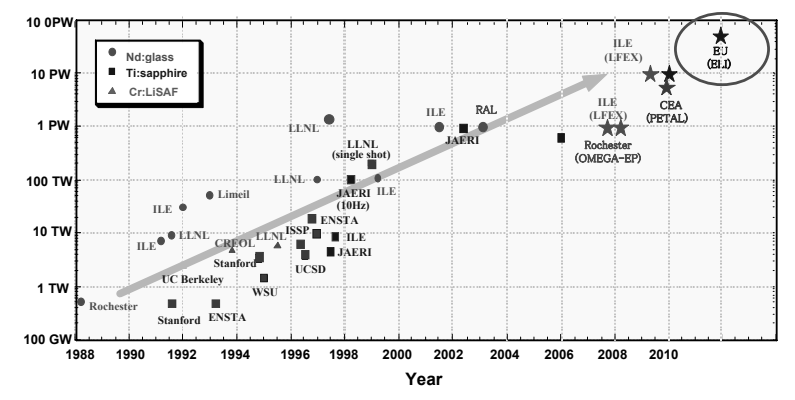

FIG. 2 Progress in ultraintense lasers by CPA techniques.

PW lasers in Japan [10]. FIREX (Fast Ignition Realization Experiment) project [11] started toward fast ignition and fusion burn. The fast ignition scheme is very attractive in the design of a laser fusion power plant because of the higher gain with smaller laser energy.

It is expected that the laser fusion ignition and energy gain of $\sim 10$ will be achieved during $2010-2013$ by the central ignition and at around 2015 by the fast ignition. A possible road map toward a laser fusion power plant is shown in Fig. 3. The next step of laser fusion research after ignition is to demonstrate electric power generation by an experimental reactor. It is important to develop new lasers with rep-rate operation which can deliver pulse energy of $10 \sim 100 \mathrm{~kJ}$ at $10 \sim 20 \mathrm{~Hz}$. For this purpose, the small-scale modules of a driver laser system, such as Mercury [12], HALNA [13, 14], Lucia [15], and Polaris [16] have been developed, as shown in Fig. 1 and Fig. 4.

\section{NEW APPLICATIONS OF HIGH POWER LASER}

3.1 High-energy density physics, laboratory astrophysics, and geophysics

By focusing a high-energy laser like the GEKKO XII, we can produce a high-energy density plasma in sub-

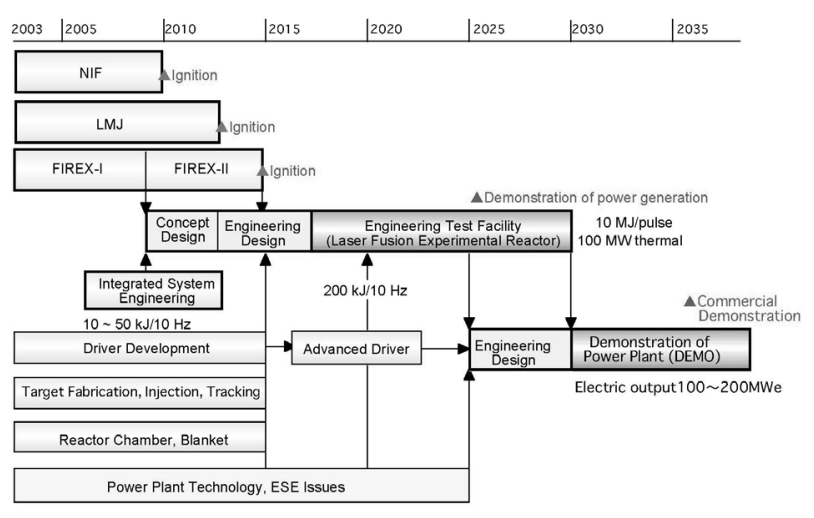

FIG. 3. Road map toward laser fusion power plant.

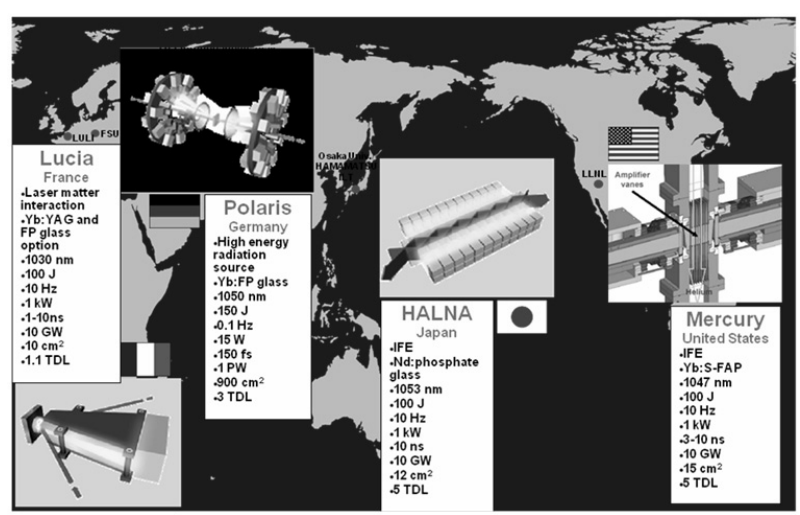

FIG. 4. High-energy diode-pumped solid-state lasers for inertial fusion energy developments.

millimeter-scale volumes. The compressed material at a density of $100 \mathrm{~g} / \mathrm{cm}^{3}$ and heated up to $100 \mathrm{eV}$ is at $10 \mathrm{Gbar}$, which corresponds to $10^{7} \mathrm{~J} / \mathrm{cm}^{3}$. Such a regime of high-energy density gives us access to phenomena in a laboratory that otherwise appear only in energetic astrophysical systems, and opens new fields of science such as high-energy density physics and laboratory astrophysics. The temperature-density parameter space for typical astrophysical phenomena and the regimes accessible by a high-energy laser are shown in Fig. 5. Studies on high Mach number jets, hydrodynamic instabilities, radiation flows, opacities and equation of state (EOS), and strong shock and compressions, which are relevant to supernovae, $\gamma$-ray burst, giant planet, interiors of Jupiter and Earth, and so on, have been performed $[17,18]$.

\subsection{Laser accelerator and laser nuclear physics}

By focusing a highly intense laser on a target, we can obtain the laser intensity over $10^{20} \mathrm{~W} / \mathrm{cm}^{2}$. Under such a super-strong field, electrons in matter are accelerated into the relativistic regime. As is well known, the so-called hot electron component, along with thermal electrons, is formed at $I \lambda^{2}>10^{14} \mathrm{W \mu m^{2 }} / \mathrm{cm}^{2}$, where $I$ is the laser intensity in $\mathrm{W} / \mathrm{cm}^{2}$ and $\lambda$ is the laser wavelength in 


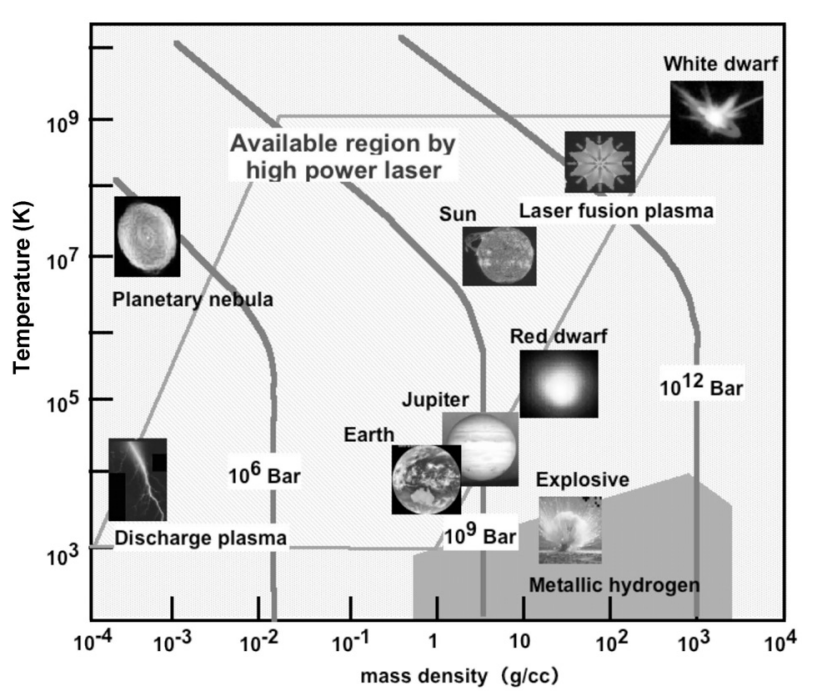

FIG. 5. Parameter space of various plasma conditions and those accessible by a high-energy laser.

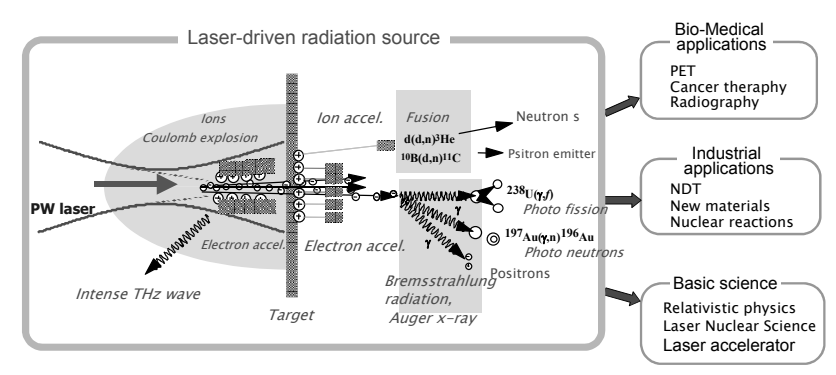

FIG. 6. Ultraintense laser-matter interactions and their applications.

$\mu \mathrm{m}$. The hot electron component has a non-Maxwellian distribution function of electron energy. The average temperature of hot electrons increases with the increase of laser intensity. At $>10^{20} \mathrm{W \mu m^{2 }} / \mathrm{cm}^{2}$, hot electron temperature reaches up to $10-15 \mathrm{MeV}$, and the maximum electron energy exceeds $\sim 100 \mathrm{MeV}$ [19]. A variety of physical mechanisms of electron accelerations have been discussed depending on the laser and target conditions [20-22].

When a gaseous target is used, monoenergetic electron beams are generated, under the particular plasma conditions, through a laser wakefield acceleration [23-25]. Recently, it was demonstrated to generate $\mathrm{GeV}$ electron beams from a cm-scale laser-plasma accelerator [26]. Laser-plasma-based accelerators have the potential to deliver an accelerating gradient more than 1000 times greater than those produced by conventional accelerator technology, and on a tabletop scale.

The generation of a collimated hot electron beam leads to the formation of an ion beam, which propagates along the normal to the target surface [21, 27]. When the hot electrons leave the target surface, a charge-separation field is formed. Field gradient reaches to the order of
$\mathrm{MeV} / \mu \mathrm{m}$. Such fields can ionize atoms and rapidly accelerate ions. Protons with the energies up to $60 \mathrm{MeV}$ and heavier ions with the energies up to $\sim 7 \mathrm{MeV}$ per nucleon have been observed [28-32]. The proton beams have a number of unique properties including high brightness $\left(\sim 10^{17}\right.$ ions in picosecond-scale bunches) and ultralow emittance [33].

Energetic electrons, protons, heavier ions, and bremsstrahlung $\gamma$-rays, emitted from a relativistic plasma, may induce nuclear processes [20, 34 - 37]. A number of experimental results on photoexcitation of nuclei, photonuclear reactions such as $(\gamma, n)$ and $(\gamma, \mathrm{x} n)$, photofission $(\gamma$, $f)$, proton and heavier ion induced nuclear reactions such as $(p, n),(p, \mathrm{x} n),(p, p+n),(p, \alpha+n)$ and $(d, n)$, fusion reactions, and so on, have been reported [19, 3841]. Laser-matter interactions in the ultra-intense laser region are summarized in Fig. 6. The laser plasma itself is a point source of energetic radiations and/or particles. A number of industrial applications of this point source have been proposed and experimentally demonstrated, which include proton imaging, proton therapy of cancer tissue, production of medical isotopes for PET, injection into large-ion accelerators, nuclear spallation, proton fast ignition for laser fusion, transmutation of high-level radioactive waste from a fission reactor, and a pulsed neutron source for various applications [21,36].

\subsection{Cancer therapy by laser-driven ion beam}

The use of protons and carbon ions in radiotherapy has several advantages to the more widely used X-ray radiotherapy. The proton beam scattering on the atomic electrons is weak, and there is less irradiation of healthy tissues in the vicinity of the tumors. The slowing-down length for a proton with a given energy is fixed, which avoids undesirable irradiation of healthy tissues at the rear side of the tumor. And the well-localized maximum of the proton energy losses in matter (Bragg peak) leads to a substantial increase of the irradiation dose in the vicinity of the proton stopping point.

The proton energy window of therapeutical interest ranges between 60 and $250 \mathrm{MeV}$, depending on the location of the tumor. The use of laser-based accelerators has been proposed as an alternative [42-46], which could lead to advantages in terms of device compactness and costs.

Typically, the proton energy of $\sim 200 \mathrm{MeV}$, the energy spread of $\sim 10^{-2}$, and the number of $10^{10}$ protons $/ \mathrm{s}$ are required for optimal dose delivery over the tumor region. In order to reach these values a new high repetition rate $\mathrm{PW}$ laser system, which can deliver $30 \mathrm{~J}, 30 \mathrm{fs}$, $100 \mathrm{~Hz},>2 \times 10^{21} \mathrm{~W} / \mathrm{cm}^{2}$ will be necessary.

\subsection{Production of PET isotopes}

Positron emission tomography (PET) is a powerful medical diagnostic and imaging technique to detect cancer tissues in the human body [47]. The PET process 
involves the patient receiving an injection of a pharmaceutical labeled with a short-lived $\beta+$ emitting source which collects in areas of high metabolic activity within the body such as tumors. The specific sites in the body can be imaged by detecting two counterpropagating $511 \mathrm{keV}$ positron-electron annihilation $\gamma$-rays emitted from the radiopharmaceutical. The principal tracers used in the PET are ${ }^{11} \mathrm{C},{ }^{13} \mathrm{~N},{ }^{15} \mathrm{O}$, and ${ }^{18} \mathrm{~F}$, halflives of which are $20.34 \mathrm{~min}, 123 \mathrm{~s}, 9.96 \mathrm{~min}$, and 109.7 min, respectively. PET isotopes are produced using energetic proton beams from cyclotrons via $(p, n)$ or $(p, \alpha)$ reactions.

One of the main factors limiting the wider use of PET imaging is the requirement for expensive infrastructure at the heart of which lies in the cyclotron and the associated extensive radiation shielding. A more simplified approach to isotope production would be to develop a miniaturized, on-site resource with eventual capability similar to that of cyclotron.

A powerful candidate of alternatives is a laser driven proton beam to produce PET isotopes. The basic experiments have been performed and the possibility for developing on-site, easy-to-shield, and compact laser system was also discussed [48-50]. For example, $10 \mathrm{MBq}$ of ${ }^{11} \mathrm{C}$ were generated via ${ }^{11} \mathrm{~B}(p, n){ }^{11} \mathrm{C}$ reaction, when a boron sample was irradiated by proton beams which were produced by irradiating $\sim 300 \mathrm{~J} / \sim 1 \mathrm{ps}$ laser on to a foil target at $2 \times 10^{20} \mathrm{~W} / \mathrm{cm}^{2}[48]$. The required activities of positron emitter for PET are $\sim 1 \mathrm{GBq}$ for ${ }^{11} \mathrm{C}$, and $\sim 0.5 \mathrm{GBq}$ for ${ }^{18} \mathrm{~F}$. It is suggested from the various experimental results given in [48-50] that, in order to achieve these activities, a compact laser system with an output energy of $10 \mathrm{~J}$ in $20 \mathrm{fs}$ at $100 \mathrm{~Hz}$ repetition and focusable intensity of $>10^{20} \mathrm{~W} / \mathrm{cm}^{2}$ will be required.

\subsection{Nuclear transmutation by laser-driven $\gamma$-ray}

One of the major problems of the nuclear energy is in the managements and disposal of high-level radioactive waste. Vitrified high-level waste can be stored for about 50 years before ultimate geological disposal. Transmutation of long-lived fission products and transuranic (TRU) waste is an option to reduce the burden of nuclear waste. Several methods have been discussed which involve transmutation by bombardment with neutrons from a reactor or a particle accelerator. The other approach is to use laser-driven high-brightness $\gamma$-rays [50].

In the disposal of nuclear waste, ${ }^{129} \mathrm{I}$ is one of the primary risk considerations due to its long half-life $(1.6 \times$ $107 \mathrm{y})$, and high radio-toxicity and mobility. ${ }^{129} \mathrm{I}$ can be photo-excited to emit a single neutron and transmutate to ${ }^{128} \mathrm{I}$ through ${ }^{129} \mathrm{I}(p, n){ }^{128} \mathrm{I}$ reaction. ${ }^{128} \mathrm{I}$ has a half-life of $25 \mathrm{~min}, 7 \%$ of which capture an electron and decay to ${ }^{128} \mathrm{Te}$. The remaining $93 \%$ undergo $\beta$-decay into stable ${ }^{128} \mathrm{Xe}$.

Two different ways to generate $\gamma$-rays by laser are proposed. One is to use bremsstrahlung $\gamma$-rays generated in a high-Z material target [52-54], and the other is to use Compton $\gamma$-rays due to the accumulated laser photons interacting with high-energy electrons [55]. For both cases, the transmutations of ${ }^{197} \mathrm{Au},{ }^{128} \mathrm{I}$ and ${ }^{129} \mathrm{I}$ to stable isotopes have been demonstrated by laser driven $\gamma$-rays.

Ledingham et al. irradiated $\mathrm{Au}$ target to generate $\gamma$ -rays by a $360 \mathrm{~J}$ laser pulse of $0.7 \mathrm{ps}$ pulse duration at $5 \times 10^{20} \mathrm{~W} / \mathrm{cm}^{2}[52]$. The iodine sample $\left(\mathrm{PbI}_{2}\right.$ with $70 \%{ }^{129} \mathrm{I}$ ) was placed behind the Au target, and $3 \times$ $10^{6}$ nuclei of ${ }^{128} \mathrm{I}$ were generated by transmutation. At $5 \times 10^{20} \mathrm{~W} / \mathrm{cm}^{2}$, the hot electron temperature was $6.4 \mathrm{MeV}$ and the energy of $\gamma$-ray generated by bremsstrahlung was rather small compared to the peak energy of giant dipole resonance $(\sim 15 \mathrm{MeV})$ in $(\gamma, n)$ reaction. Laser intensities exceeding $10^{22} \mathrm{~W} / \mathrm{cm}^{2}$ will be required for more efficient $(\gamma, n)$ reaction [53].

\subsection{Pulsed neutron source by lasers}

Neutrons are widely used in a various field of science and industry, which include neutron radiography, doping of ${ }^{31} \mathrm{P}$ into Si by NDT (Neutron Transmutation Doping) technique, cancer therapy by BNCT (Boron Neutron Capture Therapy), fusion materials research and so on. Neutron radiography is a similar technique as X-ray radiography. The difference between them is in interaction mechanism: neutrons interact with atomic nucleus, X-ray with the electronic shell. Light elements as hydrogen, lithium and boron deliver high contrast for neutrons, but are more or less transparent for X-rays. Both methods are complementary to each other.

The neutron sources, most often used, are fission reactors (continuous source) and proton accelerator driven spallation sources (pulsed source). Because of their large neutron irradiation facilities, the installations require a lot of trained personnel to operate and to maintain, and need very strict radiation shielding. Recently two kinds of laser driven pulsed neutron sources have been proposed. One is based on D-T fusion reactions [56-58], and the other is the neutron source by ${ }^{7} \mathrm{Li}(p$, $n)^{7} \mathrm{Be}$ or $\mathrm{Pb}(p, \mathrm{x} n) \mathrm{Bi}$ nuclear reaction induced by laser driven proton beams [59].

Since the beginning of laser fusion research the neutron yields generated by using large-scale high-energy lasers increased as shown in Fig. 7. Fusion neutrons from the explosion of deuterium clusters were observed with a compact Ti:sapphire laser [60]. The neutron production by $(\gamma, n)$ and $(p, n)$ reactions induced by laser driven $\gamma$-rays and proton beams were also demonstrated $[61$, 62 . For D-T fusion and proton induced nuclear reaction, $10^{9}$ neutrons $/ \mathrm{J}$ and $\sim 10^{7}$ neutrons /J have been achieved, respectively. In the applications for neutron radiography, BNCT, and fusion materials research, a neutron yield or neutron flux of $10^{11 \sim 13}$ neutrons $/ \mathrm{sr}, 10^{9}$ neutrons $/ \mathrm{cm}^{2} \mathrm{~s}$ (thermal neutrons), and $>10^{12}$ neutrons $/ \mathrm{cm}^{2} \mathrm{~s}(14 \mathrm{MeV}$ neutrons), respectively, are necessary. In order to achieve these values, lasers with the output of $\sim 1 \mathrm{~kJ} / 1-10$ $\mathrm{ps} / 100 \mathrm{~Hz}$ will be required. 


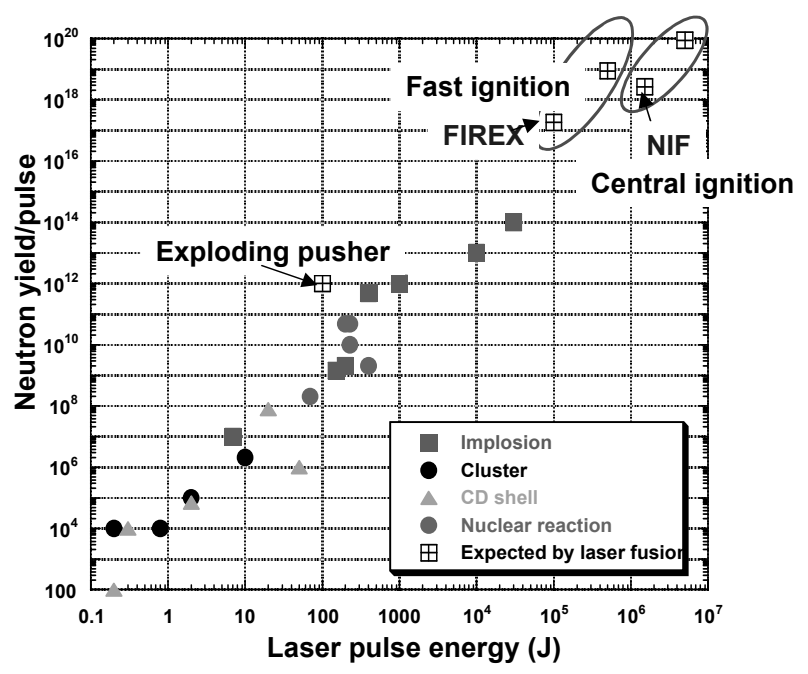

FIG. 7. Neutron yield and its laser energy scaling.

\section{ADVANCED HIGH POWER LASER BY CRYOGENIC YB:YAG and OPCPA (GENBU LASER)}

As described in Sec. III, many new industrial applications of high power lasers have been proposed. In order to realize these proposals an advanced new laser that can deliver high-energy pulse and/or highly intense pulse with high repetition rate is required to be developed. Table 1 and Figure 8 show the summary of the laser

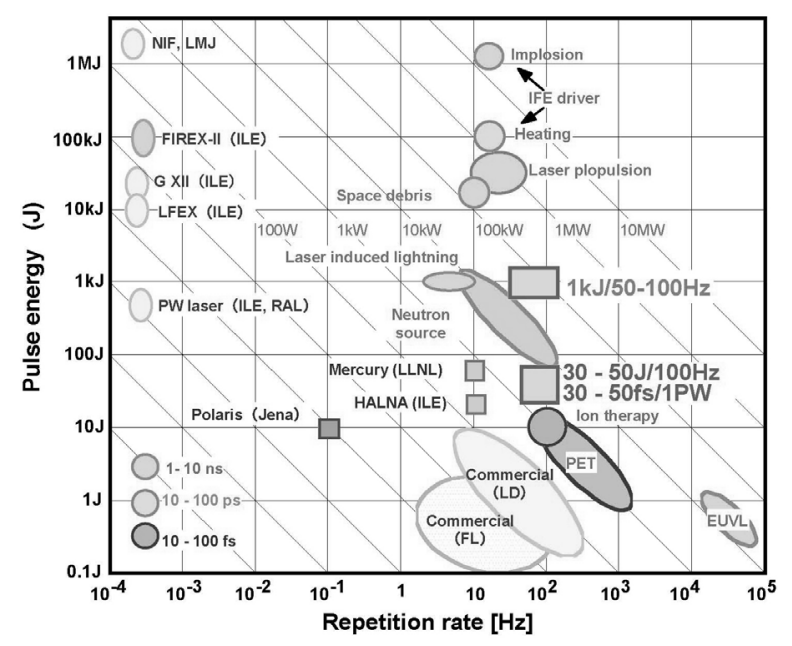

FIG. 8. Laser parameters required for new applications. Output of high power lasers, already developed and under development are superimposed.

parameters required for the new applications. In Fig. 8 , the laser pulse energy and the repetition rate, already developed and under developments, are also superimposed. It is suggested that, if a $1 \mathrm{~kJ} / 50-100 \mathrm{~Hz}$ laser and 30 - $50 \mathrm{~J} / 30$ - $50 \mathrm{fs} />1 \mathrm{PW} / 100 \mathrm{~Hz}$ laser are available, the proposed new application will be realized. We have conceptually designed a new laser system named GENBU (Generation of Energetic Beam Ultimate). The system consists of a main laser and an ultra-short pulse laser pumped by the main laser. The goals of the GENBU are to achieve $1 \mathrm{~kJ} / 10-100 \mathrm{ps} / 100 \mathrm{~Hz}$ and 30-50J/10-30

TABLE. 1. New applications and required laser parameters.

\begin{tabular}{|c|c|c|}
\hline \multicolumn{2}{|c|}{ Applications } & Requirements \\
\hline \multirow{2}{*}{$\begin{array}{c}\text { Laser fusion } \\
\text { (Fast ignition) }\end{array}$} & for implosion & $1 \mathrm{MJ} / 10 \mathrm{~ns} / 0.35 \mu \mathrm{m} / 16 \mathrm{~Hz} / 16 \mathrm{MW}$ \\
\hline & for ignition & $100 \mathrm{~kJ} / 30 \mathrm{ps} / 1 \mu \mathrm{m} / 16 \mathrm{~Hz} / 1.6 \mathrm{MW}$ \\
\hline \multirow{5}{*}{ Neutron source } & \multirow{3}{*}{$\begin{array}{l}\text { for material } \\
\text { development }\end{array}$} & $40 \mathrm{~kJ} / 10 \mathrm{~ns} / 1 \mathrm{\mu m} / 10 \mathrm{HZ} / 400 \mathrm{~kW}(\mathrm{LAHRT}$ target) \\
\hline & & $1 \mathrm{~kJ} / 15 \mathrm{ps} / 1 \mu \mathrm{m} / 10 \mathrm{HZ} / 10 \mathrm{~kW}$ (exploding pusher) \\
\hline & & $100 \mathrm{~J} / 6-60 \mathrm{ps} / 1 \mu \mathrm{m} / 100 \mathrm{~Hz} / 10 \mathrm{~kW}$ (beam fusion) \\
\hline & $\mathrm{BNCT}$ & $1 \mathrm{~kJ} / \sim 10 \mathrm{ps} / 1 \mu \mathrm{m} / 1 \mathrm{kHZ} / 1 \mathrm{MW}$ \\
\hline & Neutron radiography & $10 \mathrm{~J} / 100 \mathrm{fs} / 10-100 \mathrm{~Hz} / 10^{21} \mathrm{~W} / \mathrm{cm}^{2}$ \\
\hline \multirow{3}{*}{ Space application } & Laser propulsion & $10-50 \mathrm{~kJ} / 10 \mathrm{~ns} / 10-50 \mathrm{~Hz} / 500 \mathrm{~kW}$ \\
\hline & Space debris removal & $15 \mathrm{~kJ} / 10 \mathrm{~ns} / 0.5 \mathrm{~mm} / 2 \mathrm{kHz} / 30 \mathrm{~kW}$ \\
\hline & Energy transport & $1-10 \mathrm{MW}(\mathrm{CW})$ \\
\hline \multicolumn{2}{|c|}{ Laser-induced lightning } & $1 \mathrm{~kJ} / 10 \mathrm{~Hz} / 1-10 \mathrm{~ns} / 10 \mathrm{~kW}$ \\
\hline \multirow{4}{*}{ lon beam } & \multirow{2}{*}{$\begin{array}{l}\text { Production of positron } \\
\text { emitter for PET }\end{array}$} & $10 \mathrm{~J} / 20 \mathrm{fs} / 100 \mathrm{~Hz} / 1 \mathrm{~kW} / 10^{20} \mathrm{~W} / \mathrm{cm}^{2}$ \\
\hline & & $50 \mathrm{~J} / 1 \mathrm{~ns} / 0.5 \mu \mathrm{m} / 100 \mathrm{~Hz} / 5 \mathrm{~kW}$ (for pump) \\
\hline & \multirow{2}{*}{ Cancer therapy } & $10-30 \mathrm{~J} / 10-30 \mathrm{fs} / 10-100 \mathrm{~Hz} />2 \times 10^{21} \mathrm{~W} / \mathrm{cm}^{2}$ \\
\hline & & $50-100 \mathrm{~J} / 1 \mathrm{~ns} / 0.5 \mu \mathrm{m} / 100 \mathrm{~Hz} / 10 \mathrm{~kW}$ (for pump) \\
\hline EUV source & & $0.1-1 \mathrm{~J} / \sim 1 \mathrm{~ns} / 10-100 \mathrm{kHz} / 10-20 \mathrm{~kW}$ \\
\hline
\end{tabular}




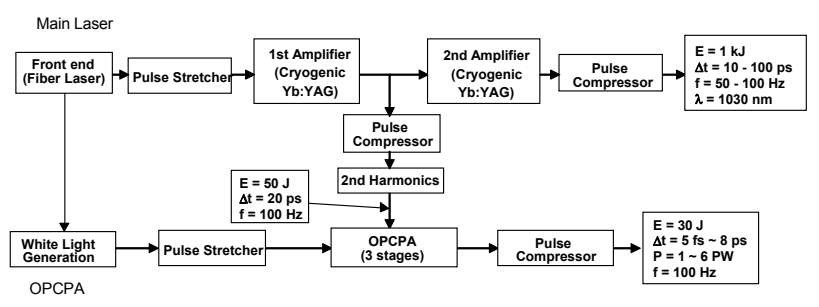

FIG. 9. Schematic of the GENBU laser. The laser consists of a main laser and an OPCPA laser.

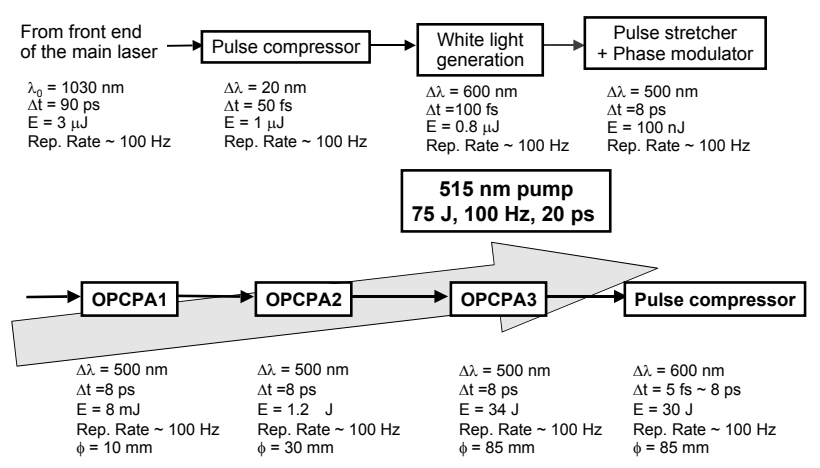

FIG. 10. Schematic of the GENBU-OPCPA chain.

fs $/ 100 H z$. In this design, a cryogenically cooled Yb:YAG and an OPCPA were selected as the main amplifier medium and ultra-short pulse amplifier, respectively $[63,64]$.

In the high-energy laser systems mentioned in Sec. II, Nd:glasses were used as laser amplifier medium because of the ability to fabricate a large sized laser medium. Recent remarkable progress in ceramic crystals made it possible to utilize them for a high-energy laser. The thermal conductivity of crystal is much larger than that of glass, and the repetitive operation of a high-energy laser by ceramic crystal will be possible.

The Yb-doped crystals such as Yb:YAG for high-energy and high rep-rate lasers have several advantages over the Nd-doped materials, which include small thermal effects due to low quantum defect and long life time of fluorescence for efficient energy storage. The disadvantages are in the quasi-three level system and small emission cross-section at room temperature operation, which require the high pump intensity of excitation pulse and the high saturation flux for efficient extraction of the stored energy. These drawbacks are improved by cooling the Yb:YAG crystal. At the temperature of about $100 \mathrm{~K}$ or less, the Yb:YAG becomes the four level system and no high pump intensity is required [65]. In addition, the emission cross-section increases because of fluorescence narrowing.

The OPCPA technique [66] is based on the direct energy conversion from the pump beam to the signal and/or idler beams in the nonlinear crystals, and is one of the most powerful techniques in the generation of high-energy and ultra-short laser pulses. Its major ad- vantages include high gain, high contrast, low heat deposition, high beam quality, and ultra-broad bandwidth [67]. The capability to use a large nonlinear crystal such as KDP and DKDP is also attractive for a high-energy laser pulse.

A schematic diagram of GENBU laser is shown in Fig. 9. The main laser consists of a front end, a pulse stretcher, two stages of Yb:YAG amplifier and a pulse compressor. A goal of the main laser is to generate an output energy of $1 \mathrm{~kJ}$ in $10 \sim 100 \mathrm{ps}$ pulse duration at $100 \mathrm{~Hz}$ repetition rate. In the amplifier chain, the chirped pulse amplification is adopted. The amplifier material is a cryogenically cooled Yb:YAG ceramics to realize the high-energy density operation, leading the system size compact. A new polygonal amplifier structure is proposed by utilizing active mirror amplifiers [69]. After the first amplifier, the laser pulse is extracted for to use as a pump source of OPCPA chain.

Figure 10 shows a schematic of the GENBU-OPCPA chain. A seed pulse is obtained from the front end of the GENBU-main laser. The pulse is focused onto a piece of fused silica to generate a white continuum. The white light is then temporally stretched and has its phase pre-compensated by a phase modulator. The pulse is amplified in the series of OPA and finally compressed. The goal of the GENBU-OPCPA is to produce a fewcycle $(\sim 5 \mathrm{fs})$ pulse with an energy of $30 \mathrm{~J}$ which corresponds to the peak power of $6 \mathrm{PW}$ at the repetition rate of $100 \mathrm{~Hz}$.

\section{SUMMARY}

Recent progresses in high power lasers and laser fusion research, and the new applications of high-energy and ultra-intense lasers are reviewed. A conceptual design of a new laser, named GENBU, was described. We have started the detailed design works and the small-scale experiments of the GENBU main laser and also OPCPA system [69-71]. We believe that these new technologies of a cryogenic Yb:YAG amplifier, a large size active mirror, and a broadband OPCPA could be one of the core technologies for the inertial fusion energy developments and also the new applications of high-energy ultraintense lasers.

\section{ACKNOWLEDGMENT}

Design works on the GENBU laser were performed under the High-Power Laser Development Committee supported by IFE Forum. We are grateful to the committee members. 


\section{REFERENCES}

[1] C. Yamanaka et al., "Laser-implosion of high-aspect-ratio targets produces thermonuclear neutron yields exceeding 1012 by use of shock multiplexing," Phys. Rev. Lett., vol. 56, no. 15, pp. 1575-1578, 1986.

[2] H. Azechi et al., "High density compression experiments at ILE, Osaka," Laser and Particle Beams, vol. 9, no. 2, pp. 193-207, 1991.

[3] E.I. Moses, "Ignition on the National Ignition Facility," J. Phys.: Conf. Series, vol. 112, p. 012003, 2008.

[4] C.A. Haynam et al., "National Ignition Facility laser performance status," Appl. Opt., vol. 46, no. 16, pp. 3276-3303, 2007.

[5] J. Ebrardt and J.M. Chaput, "LMJ project status," J. Phys.: Conf. Series, vol. 112, p. 032005, 2008.

[6] M.D. Perry et al., "Petawatt laser pulses," Opt. Lett., vol. 24, no. 3, pp. 160-162, 1999.

[7] Y. Kitagawa et al., "Prepulse-free petawatt laser for a fast ignitor," IEEE J. Quantum Electron., vol. 40, no. 3, pp. 281- 293, 2004.

[8] K. Yamakawa, " Table top lasers create ultrahigh peak powers," Oyo-Butsuri, vol. 73, no. 2, pp. 186-193, 2004 (in Japanese).

[9] http://www.extreme-light-infrastructure.eu/

[10] R. Kodama et al., "Fast-heating of ultra-high density plasma as a step toward laser fusion ignition," Nature, vol. 412, no. 6489 , pp. 798-802, 2001, R. Kodama et al., "Fast heating scalable to laser fusion ignition," Nature, vol. 418, no. 6901, pp. 933-934, 2002.

[11] H. Azechi and FIREX project, "The FIREX program on the way to inertial fusion energy," J. Phys.: Conf. Series, vol. 112, p. 012002, 2008.

[12] A. J. Bayramian et al., "Activation of the Mercury laser system: A diode-pumped solid-state laser driver for inertial fusion," Proc. Inertial Fusion Science and Applications (IFSA) 2001, pp.459-464, ed. K.A. Tanaka et al., Elsevier, 2002.

[13] T. Kawashima et al., "Design and performance of a diodepumped Nd:silica-phosphate glass zig-zag slab laser amplifier for the inertial fusion energy," Jpn, J. Appl. Phys., vol. 40, no. 11, pp. 6415-6425, 2001.

[14] R. Yasuhara et al., " $213 \mathrm{~W}$ average power of $2.4 \mathrm{GW}$ pulsed thermally controlled Nd:glass zigzag slab laser with a stimulated Brillouin scattering mirror," Opt. Lett., vol. 33, no. 15, pp. 1711-1713, 2008.

[15] S. Bahbah et al., "High power Yb:YAG diode pumped Lucia front-end oscillator (250mJ, 50ns, 2Hz), J. Phys.: Conf. Series, vol. 112, p. 032053, 2008.

[16] J. Hein et al., "Polaris: An all diode-pumped ultrahigh peak power laser for high repetition rates," in Lasers and nuclei, pp.47-66, ed. H. Schwoerer, J. Magil and B. Beleites, Springer, 2006.

[17] B. A. Remington et al., "A review of astrophysics experiments on intense lasers,” Phys. Plasmas, vol. 7, no. 5, pp. 1641-1652, 2000.

[18] M. Ikoma et al., "Unveiling the interior of Jupiter with high-power lasers: equation of state of hydrogen at several 100 GPa,” J. Plasma Fusion Res., vol. 84, no. 2, pp. 93-99, 2008 (in Japanese), K. Shigemori et al., "Measure- ment of sound velocity of laser-irradiated iron foils relevant to Earth core condition," Eur. Phys. J. D, vol. 44, pp. 301-305, 2007, K. Shigemori et al., "Multiple shock compression of diamond foils with a shaped laser pulse over 1 TPa," J. Phys.: Conf. Series, vol. 112, p. 042023, 2008.

[19] T. E. Cowan et al., "Photonuclear fission from high energy electrons from ultraintense laser-solid interactions," Phys. Rev Lett., vol. 84, no. 5, pp. 903-906, 2000.

[20] A. V. Andreev, V.M. Gordienko and A.B. Savel'ev, "Nuclear processes in a high-temperature plasma by an ultrashort laser pulse," Quantum Electron., vol. 31, no. 11, pp. 941-956, 2001.

[21] M. Borghesi et al., "Fast ion generation by high-intensity laser irradiation of solid target and applications," Fusion Sci. Technol., vol. 49, no. 4, pp. 412-439, 2006.

[22] H. Schwoere, "High-intensity laser-matter interaction," in Lasers and nuclei, pp.7-24, ed. H. Schwoerer, J. Magil and B. Beleites, Springer, 2006.

[23] S.P.D. Mangles et al., "Monoenergetic beams of relativistic electrons from intense laser-plasma interactions," Nature, vol. 431, no. 7008, pp. 535-541, 2004.

[24] C.G.R. Geddes et al., "High-quality electron beams from a laser wakefield accelerator using plasama-channel guiding," Nature, vol. 431, no. 7008, pp. 538-541, 2004.

[25] J. Faure et al., "A laser-plasma accelerator producing monoenergetic electron beams," Nature, vol. 431, no. 7008, pp. 541-544, 2004.

[26] W. P. Leemans et al., "GeV electron beams from a centimeter-scale accelerator,” Nature Phys., vol. 2, no. 10, pp. 696-699, 2006, N.A.M. Hafz et al., "Stable generation of $\mathrm{GeV}$-class electron beams from self-guided laserplasma channels," Nature Photonics, vol. 2, no. 9, pp. 971-977, 2008.

[27] V. Malka, J. Faure, S. Fritzer, and Y. Glinec, "Electron and proton beams produced by ultrashort laser pulses," in Lasers and nuclei, pp.81-90, ed. H. Schwoerer, J. Magil and B. Beleites, Springer, 2006.

[28] E. L. Clark et al., "Measurements of energetic proton transport through magnetized plasma from intense laser interactions with solids," Phys. Rev. Lett., vol. 84, no. 4, pp. 670-673, 2000.

[29] R.A. Snavely et al., "Intense high energy proton beams from petawatt-laser irradiation of solids," Phys. Rev. Lett., vol. 85, no. 14, pp. 2945-2948, 2000.

[30] S.C. Wilks et al., "Energetic proton generation in ultraintense laser-solid interactions," Phys. Plasmas, vol. 8, no. 2, 542-549, 2001.

[31] M. Hegelich et al., "MeV ion jets from short-pulse-laser interaction with thin foils," Phys. Rev. Lett. 89, 085002 (2002).

[32] P. MaKenna et al., "Proton and heavier ion acceleration in ultrahigh intensity laser-interactions with heated target fiols," Phys. Rev. E 70, 036405 (2004).

[33] L. Robson et al., "Scaling of proton acceleration driven by patawatt-laser-plasma interactions," Nature Phys., vol. 3, no. 1, pp. 58-62, 2007.

[34] H. Takabe et al., "Laser Nuclear Physics," J. Plasma Fusion Res., vol. 77, no. 11, pp. 1094-1136, 2001 (Special Topic Article).

[35] H. Takabe, "Laser Nuclear Physics," AAPPS Bulletin, 
vol. 13, no. 1, pp. 18-25, 2003.

[36] K.W.D. Ledingham, P. Mc Kenna, and R.P. Singhal, "Applications for nuclear phenomena generated by ultraintense lasers," Science, vol. 300, pp. 1107-1111, 2003.

[37] F. Ewald, "Laser-triggerd nuclear reactions," in Lasers and nuclei, pp.25-45, ed. H. Schwoerer, J. Magil and B. Beleites, Springer, 2006.

[38] M.D. Perry et al., "Hard x-ray production from high intensity laser solid interactions," Rev. Sci. Instrum., vol. 70, no. 1, pp. 265-269, 1999.

[39] K.W.D. Ledingham et al., "Photonuclear physics when multiterawtt laser pulse interacys with solid targets," Phys. Rev. Lett, vol. 84, no. 5, pp. 899-902, 2000.

[40] H. Schwoerer et al., "MeV X rays and photoneutrons from femtosecond laser-produced plasmas," Phys. Rev. Lett., vol. 86, no. 11, pp. 2317-2320, 2001.

[41] P. McKenna et al., "Broad energy spectrum of laseraccelerated protons for spallation-related physics," Phys. Rev. Lett., vol. 94, p. 084801, 2005.

[42] S.V. Bulanov et al., "Oncological hadrontherapy with laser ion accelerators,” Phys. Lett. A, vol. 299, no. 2-3, pp. 240-247, 2002.

[43] S.V. Bulanov and V.S. Khoroshkov, "Feasibility of using laser ion accelerators in proton therapy," Plasma Phys. Rep., vol. 28, pp. 453-457, 2002.

[44] E. Foulkal and C. Ma, "Laser accelerated carbon ion beams for radiation therapy,” Med. Phys., vol. 30, p. 1448, 2003.

[45] V. Malka et al., "Practicability of protontherapy using compact laser systems," Med. Phys., vol. 31, no. 6, pp. 1587-1592, 2004.

[46] E. Foulkal et al., "Energy and intensity modulated radiation therapy using laser accelerated proton beams," Med. Phys., vol. 31, p. 1884, 2004.

[47] L. Robson et al., "High-power laser production of PET isotopes," in Lasers and nuclei, pp.191-204, ed. H. Schwoerer, J. Magil and B. Beleites, Springer, 2006.

[48] M.I.K. Santala et al., "Production of radioactive nuclides by energetic protons generated from intense laser-plasma interactions," Appl. Phys. Lett., vol 78, no. 1, pp. 19-21, 2001.

[49] S. Fritzler et al., "Proton beams generated with highintensity lasers: Applications to medical isotope production,” Appl. Phys. Lett., vol. 83, no. 15, pp. 3039-3041, 2003.

[50] K.W.D. Ledingham et al., "High power laser production of short-lived isotopes for positron emission tomography," J. Phys. D: Appl. Phys., vol. 37, pp. 23412345, 2004.

[51] D. Li, K. Imasaki, and M. Aoki, "Analysis on coupling gamma-ray to nuclear giant resonance,” J. Nucl. Sci. Technol., vol. 39, no. 11. pp. 1247-1249, 2002.

[52] K.W.D. Ledingham et al, "Laser-driven photo-transmutation of 129I - a long-lived nuclear waste product," J. Phys. D: Appl. Phys., vol. 36, pp. L79-L82, 2003.

[53] J. Magil et al., "Laser transmutation of iodine-129," Appl. Phys. B, vol. 77, pp. 387-390, 2003.

[54] J. Magil, J. Galy, and T. Zagar, "Laser transmutation of nuclear materials," in Lasers and nuclei, pp.131-146, ed. H. Schwoerer, J. Magil and B. Beleites, Springer,
2006.

[55] K. Imasaki et al., "High brightness $\gamma$-ray generation and nuclear transmutation," in Lasers and nuclei, pp. 147-168, ed. H. Schwoerer, J. Magil and B. Beleites, Springer, 2006.

[56] L.J. Perkins et al., "The investigation of high intensity laser driven micro neutron sources for fusion materials research at high fluence", Nuclear Fusion, vol. 40, no. 1, pp. 1-19, 2001.

[57] A. Taylor et al., "A route to the brightest possible neutron source?,” Science, vol. 315, pp. 1092-1095, 2007.

[58] S. Nakai, "Development of integrated IFE system and its industrial applications as intense neutron source," J. Phys.: Conf. Series, vol. 112, p. 042070, 2008.

[59] T. Zagar, J. Galy, and J. Magil, "Pulsed neutron sources by table-top accelerated protons," in Lasers and nuclei, pp. 109-128, ed. H. Schwoerer, J. Magil and B. Beleites, Springer, 2006.

[60] T. Ditmire et al., "Nuclear fusion from explosions of femtosecond laser-heated deuterium clusters," Nature, vol. 398, pp. 489-492, 1999.

[61] J.M. Yang et al., "Neutron production by fast protons from ultraintense lase-plasma interactions," J. Appl. Phys., vol. 96, no. 11, pp. 6912-6918, 2004.

[62] K.L. Lancaster et al., "Characterization of $7 \mathrm{Li}(\mathrm{p}, \mathrm{n}) 7 \mathrm{Be}$ neutron yields from laser produced ion beams for fast neutron radiography," Phys. Plasmas, vol 11, no. 7, pp. 3404-3408, 2004.

[63] J. Kawanaka et al., "Generation of energetic beam ultimate (GENBU) laser -main laser-," Technical digest of APLS 2008, p. 56, Nagoya, Jan. 2008.

[64] K. Yamakawa et al., "Generation of energetic beam ultimate (GENBU) laser -OPCPA laser-," Technical digest of APLS 2008, p. 57, Nagoya, Jan. 2008.

[65] S. Tokita et al., "Sapphire-conductive end-cooling of high power cryogenic Yb:YAG lasers," Appl. Phys. B, vol. 80, pp. 635-638, 2005.

[66] A. Dubietis et al., "Powerful femtosecond pulse generation by chirped and stretched pulse parametric ampkification in BBO crystal," Opt. Commun., vol. 88, pp. 437-440, 1992.

[67] H. Yoshida et al., "High-power and high-contrast optical parametric chirped pulse amplification in $\beta-\mathrm{BaB}_{2} \mathrm{O}_{4}$ crystal," Opt. Lett., vol. 28, no. 4, pp. 257-259, 2003.

[68] J. Kawanaka et al., "New concept for laser fusion energy driver by using cryogenically-cooled Yb:YAG ceramic," J. Phys.: Conf. Series, vol. 112, p. 032058, 2008.

[69] K. Ogawa et al., "Multi-millijoule, diode pumped, cryogenically-cooled Yb:KY(WO4)2 chirped-pulse regenerative amplifier," Opt. Exp., vol. 15, no. 14, pp. 85988602, 2007.

[70] K. Yamakawa et al., "Ultra-broadband optical parametric chirped-pulse amplification using an Yb:LiYF4 chirped pulse amplification pump laser," Opt. Exp., vol. 15, no. 8, pp. 5018-5023, 2007.

[71] Y. Akahane et al., "High-energy, diode-pumped, picosecond $\mathrm{Yb}$ :YAG chirped pulse regenerative amplifier for pumping optical parametric chirped-pulse amplification," Opt. Lett., vol. 32, no. 13, pp. 1899-1901, 2007. 